\title{
Staphylococcus aureus bei Diabetes: persistierende Fußulzerationen
}

\author{
Svea Sachse, Martin Rindert, Olaf Bach, Wolfram Kluge, Bettina Löffler
}

\section{Einleitung}

Diabetische Fußulzerationen (DFU) weisen eine Prävalenz von $2-10 \%$ der diabetischen Gesamtbevölkerung auf. Durch veränderte Lebenseinstellungen, die mit einem ungesunden Ernährungsverhalten und einer mangelnden „Walkability“ einhergehen, wird mit einer jährlich steigenden Zunahme von diabetischen Patienten zu rechnen sein. Dies beinhaltet auch einen Anstieg der Patienten, die wegen diabetischer Fußulzerationen behandelt werden müssen. Gemäß einer europäischen Studie entwickeln ca. 58\% der Patienten eine diabetische Fußinfektion (DFI). Fußulzerationen und insbesondere Infektionen sind schwer zu therapieren und daher der häufigste Grund für eine Krankenhauseinweisung bei Diabetikern. Die Pathogenese ist komplex und schließt mehrere Faktoren, wie Mikrozirkulationsstörung, periphere Neuropathie, Fußdeformationen und ein durch den Diabetes geschwächtes Immunsystem, ein. Neuropathische Patienten entwickeln häufiger eine periphere arterielle Verschlusskrankheit (pAVK), die wiederum mit einem erhöhten Risiko für eine nicht traumatische Amputation korreliert. Insgesamt liegt die Wahrscheinlichkeit einer Amputation für Patienten mit chronischen Fußulzerationen 15- bis 40-fach höher als die der Normalbevölkerung, wobei Männer unabhängig der ethnischen Herkunft ein erhöhtes Risiko tragen. Bei Frauen wird eine erhöhte peri- und postoperative Mortalität nach Amputationen beschrieben [1].

\section{Hauptteil}

\section{Das Mikrobiom bei Fußulzerationen}

Gestörte Mikrozirkulation und reduzierte Schmerzwahrnehmung begünstigen stark das Entstehen von Ulzerationen und bakteriellen Infektionen [3]. Kleinste Eintrittswunden, z. B. in Form von Druckstellen, verbleiben von den Patienten unbemerkt zum einen durch die stark geminderte Sensibilität und zum anderen durch die oft fehlenden Anzeichen einer Inflammation wie Rubor, Tumor, Dolor und Calor. Dadurch werden die Patienten häufig erst in einem fortgeschrittenen Infektionsprozess bei einem Arzt vorstellig. Die Ausbildung der Infektion kann sehr variieren ( $\bullet$ Abb. 1 ). Die Behandlung wird somit oft erst in einem späten Stadium eingeleitet. Durch die Grundkrankheit Diabetes wird die Immunantwort des Pa- tienten geschwächt, beispielsweise wird die Phagozytose der Immunzellen beeinträchtigt. Zudem besteht zumeist eine schlechte Durchblutung, sodass hämatogene Faktoren des Immunsystems den Ort des Infektionsgeschehens nur schwer erreichen können. Die verminderte Immunabwehr bedingt eine schnelle Kolonisation, Invasion und Proliferation von mikrobiellen Keimen auf und in der Wunde. Kulturell wird S. aureus als einer der häufigsten Erreger in diabetischen Fußulzerationen nachgewiesen [5]. Molekularbiologisch findet man jedoch verschiedene Bakterienspezies, die sich sowohl aus aeroben, anaeroben als auch aus opportunistischen oder aus Keimen der normalen Hautflora zusammensetzen. Oftmals bilden verschiedene Erreger einen Biofilm, welcher den Bakterien Schutz bietet. Nicht nur stark virulente Keime wie S. aureus, sondern auch niedrig virulente Bakterien wie Anaerobier, Corynebacterium ssp. oder koagulasenegative Staphylokokken, die häufig Bestandteil der normalen Hautflora sind, sind somit möglicherweise an der Ausbildung einer Infektion beteiligt. Breitet sich die Infektion bis zum Knochen aus, kann eine Osteitis entstehen, deren Prävalenz bei diabetischen Fußulzerationen mit 10-20\% beziffert wird. Wenn eine antibiotische Therapie versagt, steigt das Risiko einer Fuß- oder Beinamputation („Major Amputation“), was einen schwerwiegenden Eingriff für den Patienten und einen Verlust an Lebensqualität darstellt.

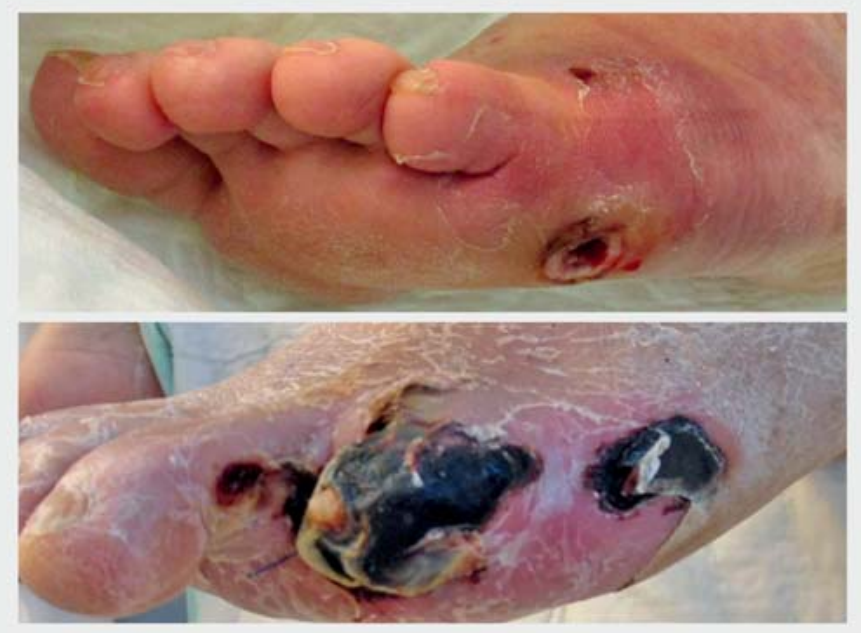

Abb. 1 Infizierte diabetische Fußulcerationen. 


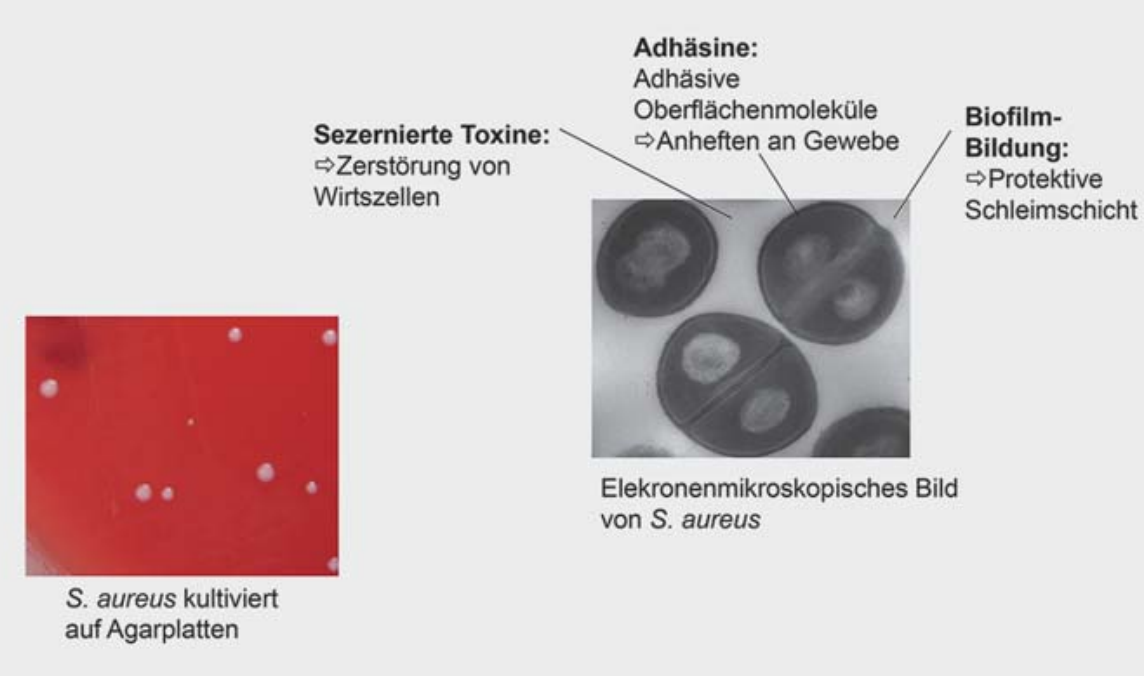

- Abb. 2 A S. aureus in verschieden großen Koloniephänotypen auf Blutagarplatten. Die großen Kolonien sind Wild-Typ-Phänotypen, die einen aktiven Stoffwechsel und Virulenzfaktorexpression aufweisen. Die kleinen Kolonien sind „small-colony-variant“(SCV-)Phänotypen, die langsam wachsen, einen reduzierten Stoffwechsel aufweisen und wenig sezernierte Virulenzfaktoren freisetzten. B Elektronenmikroskopisches Bild von sich teilenden S.-aureus-Bakterien und schematische Darstellung wichtiger Virulenzfaktoren. Adhäsine sind adhäsive Oberflächenmoleküle, die eine Anheftung der Bakterien an Wirtsstrukturen ermöglichen und die Bildung von Biofilm unterstützen, wohingegen Toxine Wirtsgewebe zerstören können.

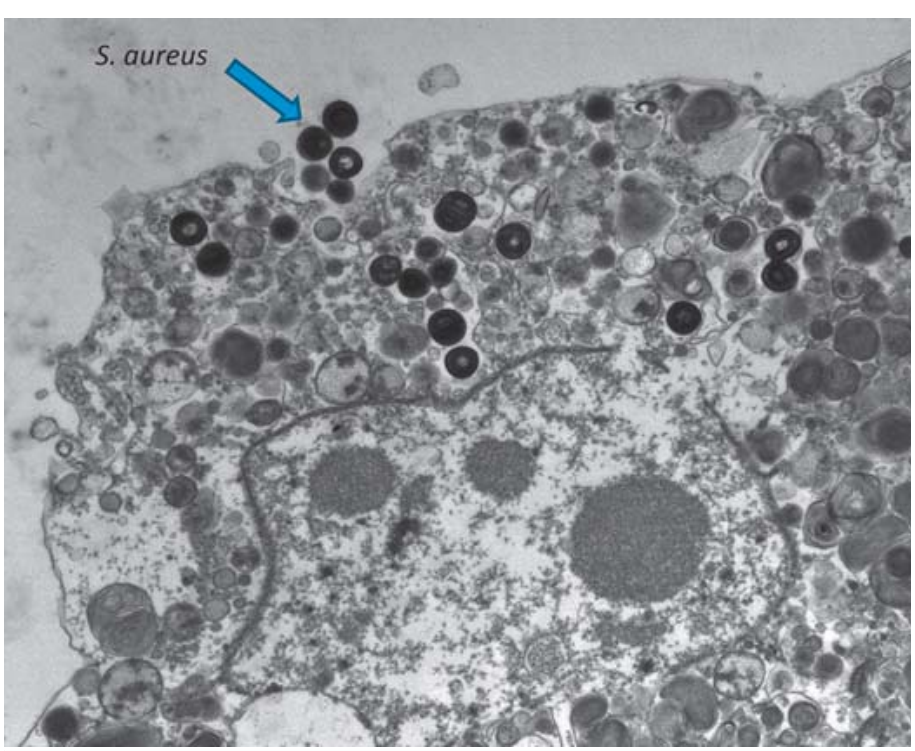

Abb. 3 Invasion von S. aureus in Wirtszellen. S. aureus kann mittels Adhäsinen an Wirtszelloberflächen anheften. Darauf folgt eine Aufnahme der Bakterien in die Wirtszelle und eine intrazelluläre Lokalisation von S. aureus. Diese Bakterienaufnahme ist ein aktiver Prozess der Wirtszelle, der für verschiedene Zelltypen, wie Endo- und Epithelzellen, aber auch Fibro- und Osteoblasten beschrieben wurde.

\section{Staphylococcus aureus: von der Kolonisation zur Infektion}

In zahlreichen Studien zu infizierten, diabetischen Fußulzerationen wurde $S$. aureus als häufigstes ätiologisches Pathogen identifiziert [5]. S. aureus ist ein fakultativ pathogener Mikroorganismus, der häufig den Nasenrachen- raum und die Haut kolonisiert. Etwa ein Drittel der erwachsenen Bevölkerung ist mit $\mathrm{S}$. aureus besiedelt, ohne Krankheitszeichen aufzuweisen. Es ist jedoch auch bekannt, dass kolonisierende S.-aureus-Stämme die Quelle für eine Infektion darstellen können, wie bei Blutstrominfektionen und Gewebeinfektionen bereits beschrieben wurde [10].

\section{Staphylococcus-aureus-Virulenz und das Entstehen von chronischen Infektionen}

S. aureus kann im Körper fast jedes Organ infizieren und dabei schwere invasive Infektionen verursachen. Um eine Infektion zu etablieren, verfügt $\mathrm{S}$. aureus über eine Vielzahl von Virulenzfaktoren, die eine Adhäsion, Invasion und Immunevasion und/oder eine Zellzerstörung verursachen ( $\bullet$ Abb. 2) [6]. Adhäsine sind eine Gruppe bakterieller Oberflächenmoleküle, welche das Anheften der Bakterien an Wirtsstrukturen vermitteln. Diese Adhärenz stellt einen ersten wichtigen Schritt im Infektionsgeschehen dar. Weiterhin sezerniert $\mathrm{S}$. aureus eine Vielzahl von Toxinen, die Wirtsgewebe zerstören können und das Eindringen der Bakterien in tiefe Gewebeschichten ermöglichen. In der aktuellen Wissenschaft wird S. aureus zunehmend als intrazelluläres Pathogen wahrgenommen, da die Bakterien in verschiedene Wirtszellen eindringen und dort für lange Zeit verbleiben können ( $\triangleright$ Abb. 3). Während einer langen intrazellulären Persistenz regulieren die Bakterien ihre Adhärenz hoch und ihre Toxinproduktion als auch den Stoffwechsel herunter und treten in eine Art "Schlafzustand“ ein ( $\bullet$ Abb. 4). Dadurch werden sie von dem Immunsystem des Wirtes mangelhaft erkannt. Der herunterregulierte Stoffwechsel hilft den Bak- 
- Tab. 1 Einteilung der Schweregrade nach Wagner/Armstrong [4].

\begin{tabular}{|c|c|c|c|c|c|c|}
\hline & 0 & 1 & 2 & 3 & 4 & 5 \\
\hline A & $\begin{array}{l}\text { prä- oder post- } \\
\text { ulzerative Läsion }\end{array}$ & $\begin{array}{l}\text { oberflächliche } \\
\text { Wunde }\end{array}$ & $\begin{array}{l}\text { Wunde bis zur } \\
\text { Ebene von Sehne } \\
\text { oder Kapsel }\end{array}$ & $\begin{array}{l}\text { Wunde bis zur } \\
\text { Ebene von Kno- } \\
\text { chen oder Gelenk }\end{array}$ & $\begin{array}{l}\text { Nekrose von } \\
\text { Fußteilen }\end{array}$ & $\begin{array}{l}\text { Nekrose des } \\
\text { gesamten Fußes }\end{array}$ \\
\hline B & mit Infektion & mit Infektion & mit Infektion & mit Infektion & mit Infektion & mit Infektion \\
\hline C & mit Ischämie & mit Ischämie & mit Ischämie & mit Ischämie & mit Ischämie & mit Ischämie \\
\hline D & $\begin{array}{l}\text { mit Ischämie und } \\
\text { Infektion }\end{array}$ & $\begin{array}{l}\text { mit Ischämie und } \\
\text { Infektion }\end{array}$ & $\begin{array}{l}\text { mit Ischämie und } \\
\text { Infektion }\end{array}$ & $\begin{array}{l}\text { mit Ischämie und } \\
\text { Infektion }\end{array}$ & $\begin{array}{l}\text { mit Ischämie und } \\
\text { Infektion }\end{array}$ & $\begin{array}{l}\text { mit Ischämie und } \\
\text { Infektion }\end{array}$ \\
\hline
\end{tabular}

Akute Infektion

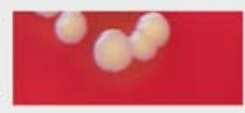

Wild-Typ-Phänotypen; Große Kolonien

Toxins $\uparrow$
Adhesins $\downarrow$
Viele Bakterien sind in
Wirtszellen/ Gewebe
eingedrungen
- Zerstörung von Gewebe durch
Toxinfreisetzung
Bakterielles Eindringen in
tiefe Gewebeschichten

Chronische Infektion
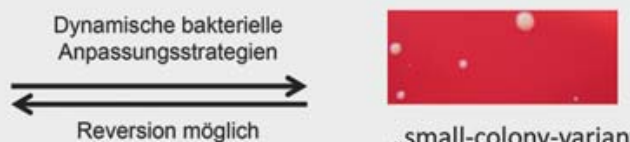

„Small-colony-variants" (SCVs); kleine Kolonien

$$
\text { Toxins ! }
$$

Adhesins $\uparrow$

- Wenige Bakterien überleben in Wirtszellen/ Gewebe

- Die Bakterien gehen durch Anpassungsstrategien in einen "Schlafzustand" über und bilden SCVs

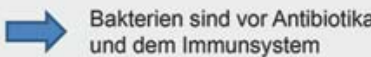
und dem Immunsystem geschützt

- Abb. 4 Schematisches Entstehen einer persistierenden Gewebeinfektion. In der akuten Phase der Infektion liegen die Bakterien überwiegend als Wild-Typ-Phänotypen vor, die viele Toxine freisetzten, Gewebe zerstören und dadurch in tiefe Gewebeschichten eindringen können. In der chronischen Phase der Infektion sind die Bakterien in Wirtsgewebe und Zellen eingedrungen und bilden SCV-Phänotypen, die durch ihren verlangsamten Stoffwechsel weitgehend vor dem Immunsystem und Antibiotikatherapien geschützt sind.

terien auch, Antibiotikatherapien besser zu überleben [8]. Diese raffinierten Anpassungsstrategien ermöglichen es S. aureus, einerseits sehr lange in Zellen bzw. Gewebe des Wirtes zu überleben und andererseits auch durch dynamische Hochregulation des Stoffwechsels immer wieder erneut einen Schub einer Infektion auszulösen.

\section{Merke}

Aufgrund dieser dynamischen bakteriellen Anpassungsstrategien ist es sehr schwierig, bei invasiven Gewebe- und Knocheninfektionen S. aureus vollständig zu eliminieren.

\section{Mikrobiologische Diagnose}

Die Standardmethode zum Nachweis von Keimen ist noch immer die Kultur, doch gibt es seit Kurzem auch kulturunabhängige, molekularbiologische Verfahren, die ein umfassenderes Bild liefern. Hier zeigte sich, dass wahrscheinlich weit mehr Mikroorganismen als bisher ange- nommen bei Fußulzerationen ätiologisch infrage kommen. Dies schließt unter anderem auch schwer bzw. nicht kultivierbare Bakterien ein. Inwieweit diese Mikroorganismen interagieren und zur Progression einer Ulzeration beitragen, muss in zukünftigen Forschungsarbeiten untersucht werden. Es ist jedoch bekannt, dass bei polymikrobiellen Infektionen zwischen den Mikroorganismen Synergien bestehen, die ein Etablieren und das Überleben niedrig virulenter Keime begünstigen [9].

\section{Verlauf und Therapie von diabetischen Fußulzerationen}

Diabetische Fußläsionen werden mittels unterschiedlicher Klassifikationssysteme gemäß ihrem Schweregrad eingeteilt ( $\bullet$ Tab. 1, Wagner-Armstrong-Klassifikation). Diese ermöglichen die interdisziplinäre Verständigung und bilden so die Basis einer fundierten und selektiven Behandlungsstrategie [1]. Jedoch ist bisher noch kein Scoring-System als Goldstandard zum Erstellen einer Ver- 
laufsprognose akzeptiert [4]. Es gilt, bei der Aufnahmeuntersuchung die oberflächlichen, infizierten Defekte von den tiefen zu unterscheiden. Bei Letzteren kann eine Phlegmone (Infektion des umgebenden Weichteilmantels), ein Defekt bis zu den darunterliegendem knöchernen Strukturen oder eine Entzündungshöhle (Abszess) vorliegen. Die Prävention gewinnt vor dem Hintergrund, dass eine Progression vom DFU zum DFI in $85 \%$ der Amputationsfälle vorliegt, an wesentlicher Bedeutung. Besteht jedoch eine infizierte Fußulzeration, so basiert die Therapie zum einen auf der antibiotischen und zum anderen auf der chirurgischen Intervention [5].

\section{Antibiotische Therapie}

Bei nicht bekanntem Keimspektrum gilt es, die Infektion über ein breit wirksames Antibiotikum, ggf. in Kombination mit einer 2. Substanzklasse, zu bekämpfen [7]. Zum Aufnahmezeitpunkt wird ein Abstrich gewonnen, wobei nach Eingang dieses Ergebnisses ggf. die Antibiose abgeändert wird. Die Antibiose wird anfangs hoch dosiert und intravenös verabreicht, um sie dann im weiteren Verlauf auf orale Medikamente umzustellen. Die Zeitdauer der antibiotischen Therapie richtet sich nach dem Verlauf der Therapie und hängt wesentlich davon ab, ob die Infektion auf den Knochen übergegriffen hat und ob die betroffenen Knochen chirurgisch entfernt werden konnten oder nicht.

\section{Chirurgische Therapie}

Direkt zur Aufnahmeuntersuchung werden die infizierten Nekrosen entfernt. Wenn eine Abszesshöhle vorhanden ist, muss diese entlastet, gespült und ggf. mehrzeitig saniert werden. Im weiteren Verlauf ist - sofern erforderlich - die Durchblutungssituation zu verbessern, damit zum einen die systemisch angewandten Antibiotika an den Ort der Infektion kommen und zum anderen nach chirurgischer Sanierung auch eine Wundheilung einsetzt [2]. Die weitere Diagnostik im Vorfeld einer chirurgischen Sanierung besteht aus einer Röntgenaufnahme (Ausmaß der Auflösung des Knochens im Sinne einer Osteitis, Störung der Statik des Fußes) und einem MRT (Weichteilinfektion, Abgrenzung zu einem „Charcot-Fuß“). Ist die Infektion auf den Knochen übergegangen und liegt somit eine Osteitis vor, ist oftmals die Resektion des betroffenen Knochens für die Heilung notwendig. Die definitive chirurgische Sanierung muss neben der Statik des Fußes auch die Möglichkeit der Deckung des Defekts mit einem belastbaren Weichteilmantel berücksichtigen. Neben den ablativen Maßnahmen (Amputationen) sind im weiteren Verlauf plastische Deckungen mit Spalthaut oder einer Lappenplastik möglich.

Merke

Ziel ist der Erhalt von möglichst viel belastbarem Fuß unter sicherer Entfernung des untergegangenen und entzündeten Gewebes.
Hier sind die Lebenssituation und der Anspruch an die resultierende Mobilität zu berücksichtigen. Die oft kardiopulmonal stark vorbelasteten Patienten profitieren nicht immer vom mehrzeitigen Vorgehen der Grenzzonenamputation.

\section{Chronischer Verlauf bei vielen Patienten}

Leider befindet sich ein Großteil der Patienten bereits seitens der Grunderkrankung in einem weit fortgeschrittenen Stadium. Auch nach stattgehabter Grenzzonenamputation und erfolgreicher Wundheilung werden sie wiederholt vorstellig. Inwieweit das Problem durch die weitere Anwendung von inadäquatem Schuhwerk oder durch in situ verbliebenen Bakterien aufrechterhalten wird, ist oft nicht hinreichend zu klären. In den meisten Fällen findet sich jedoch der ätiologische Keim des Erstbefunds wieder, sodass bei der erneuten Aufnahmeuntersuchung mit dem Antibiotikum der letzten Resistenzbestimmung begonnen wird. Oft sind die zum Operationszeitpunkt aufgedehnten Blutgefäße erneut verschlossen. Es ist allerdings nachgewiesen, dass auch eine nur temporäre Verbesserung der Durchblutung die Wundheilung ausreichend verbessert. Ansonsten gelten die gleichen Algorithmen wie bei der Erstvorstellung, wobei mit dem Patienten nach mehreren Eingriffen und lang dauernden Krankenhausaufenthalten über die Möglichkeit einer Majoramputation (Amputation oberhalb des Sprunggelenks) kritisch diskutiert werden muss.

\section{Schlussfolgerung}

Diabetische Fußulzerationen können sich schnell durch mangelnde Durchblutung und Neuropathie mit Bakterien infizieren. Kulturell wird S. aureus als einer der häufigsten Keime nachgewiesen. S. aureus besitzt durch eine Vielzahl an Virulenzfaktoren die Fähigkeit, in Gewebe und auch in Knochen vorzudringen. Dort kann er persistieren und periodisch zu neuen Infektionsausbrüchen führen. Dieses Phänomen gestaltet bei DFI die therapeutischen Maßnahmen, insbesondere die Antibiotikatherapie, schwierig. Die chirurgische Intervention unterstützt die antimikrobielle Therapie, indem einerseits die infizierten Gewebe- und Knochenteile entfernt werden und zum anderen eine Revaskularisierung erreicht wird.

\footnotetext{
Merke

Trotzdem bleibt primär die interdisziplinäre Prävention, die Aufklärung, Podologen und Internisten einschließt, als Priorität zur Vermeidung einer Fußulzeration.

Des Weiteren wird durch aktuelle Forschungsprojekte das persistierende Verhalten von S. aureus und anderen Keimen untersucht. Mit dem Wissen um die Persistenz können neue therapeutische Strategien entwickelt wer-
} 
den, die zur Reduzierung von Rezidiven, vielleicht sogar zu deren Verhinderung, führen können.

\section{Interessenkonflikt}

Die Autoren geben an, dass kein Interessenkonflikt besteht.

\section{Über die Autoren}
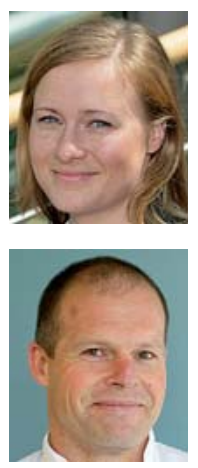

\section{Martin Rindert}

Klinik für Orthopädie, Unfall- und Handchirurgie, Sophien-Hufeland-Klinikum Weimar

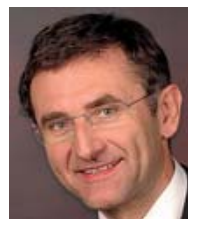

\section{Olaf Bach}

PD Dr., Klinik für Orthopädie, Unfall- und Handchirurgie, Sophien-Hufeland-Klinikum Weimar

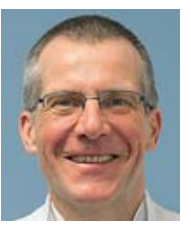

\section{Wolfram Kluge}

PD Dr., Klinik für Orthopädie, Unfall- und Handchirurgie, Sophien-Hufeland-Klinikum Weimar

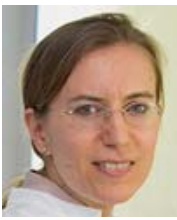

\section{Bettina Löffler}

Prof. Dr., Institut für Medizinische Mikrobiologie des Universitätsklinikums Jena
Prof. Dr. Bettina Löffler

Institut für Medizinische Mikrobiologie des Universitätsklinikums Jena

Am Klinikum 1

07747 Jena

Bettina.Loeffler@med.uni-jena.de

\section{Literatur}

[1] Armstrong DG, Lavery LA, Harkless LB. Validation of a diabetic wound classification system. The contribution of depth, infection, and ischemia to risk of amputation. Diabetes Care 1998; 21: 855-859

[2] Armstrong DG, Lavery LA, Vazquez JR et al. How and why to surgically debride neuropathic diabetic foot wounds. J Am Podiatr Med Assoc 2002; 92: 402-404

[3] Boulton AJ, Vileikyte L, Ragnarson-Tennvall G et al. The global burden of diabetic foot disease. Lancet 2005; 366: 1719-1724

[4] Frykberg RG, Zgonis T, Armstrong DG et al. Diabetic foot disorders. A clinical practice guideline (2006 revision). J Foot Ankle Surg 2006; 45 (Suppl. 5): S1-S66

[5] Lipsky BA, Berendt AR, Deery HG et al. Diagnosis and treatment of diabetic foot infections. Clin Infect Dis 2004; 39: 885-910

[6] Lowy FD. Staphylococcus aureus infections. N Engl J Med 1998; 339: 520-532

[7] Nelson EA, O'Meara S, Golder S et al. Systematic review of antimicrobial treatments for diabetic foot ulcers. Diabet Med 2006; 23: 348-359

[8] Proctor RA, Peters G. Small colony variants in staphylococcal infections: diagnostic and therapeutic implications. Clin Infect Dis 1998; 27: 419-422

[9] Smith K, Collier A, Townsend EM et al. One step closer to understanding the role of bacteria in diabetic foot ulcers: characterising the microbiome of ulcers. BMC Microbiol 2016; 16: 54

[10] Von Eiff C, Becker K, Machka K et al. Nasal carriage as a source of Staphylococcus aureus bacteremia. Study Group. N Engl | Med 2001; 344: 11-16

Bibliografie

DOI https://doi.org/10.1055/s-0043-102323

OP-JOURNAL 2017; 33: 173-177 @ Georg Thieme Verlag KG Stuttgart · New York ISSN 0178-1715 